\title{
Differences in Gastrocnemius Muscle Architectural Properties between Child Female Athletes with Different Flexibility Training Backgrounds ${ }^{\dagger}$
}

\author{
Ioli Panidi, Gregory C. Bogdanis, Vasiliki Gaspari, Polyxeni Spiliopoulou, Anastasia Donti, \\ Gerasimos Terzis and Olyvia Donti * \\ Sports Performance Laboratory, School of Physical Education and Sport Science, National and Kapodistrian \\ University of Athens,17237, Athens, Greece \\ * Correspondence: odonti@phed.uoa.gr \\ + Presented at the 9th Greek Conference of Biochemistry and Physiology of Exercise, Thessaloniki, Greece, \\ 18-20 October 2019.
}

Published: 30 August 2019

\begin{abstract}
AIM: The mechanisms underpinning long-term changes in muscle architectural characteristics and joint range of motion (ROM) following static stretching in humans remain under question, and data are sparse for growing athletes. It is possible that the characteristics of the stretching protocols used in previous training studies were not adequate to induce significant changes. An alternative approach would be to compare populations with different chronic flexibility training backgrounds. Thus, the purpose of this study was to examine differences in gastrocnemius medialis (GM) architectural characteristics at rest and during 1 min of static stretching between child athletes with different flexibility training backgrounds. MATERIAL \& METHOD: Ten female rhythmic gymnasts (RG; age. $9.0 \pm 0.7$ years) were compared to six volleyball athletes (VA; age, 9.0 \pm 0.6 years). Fascicle length, pennation angle and muscle thickness at the medial and distal part of GM, and ankle ROM were measured at rest and during $1 \mathrm{~min}$ of static stretching using ultrasonography. Data were analysed using two-way ANOVA for repeated measures on two factors (time $x$ sport). RESULTS: At rest, RG displayed similar fascicle length compared to VA at the medial $(4.19 \pm 0.37$ vs. $4.24 \pm 0.54 \mathrm{~cm}$, respectively, $p=0.841)$ and the distal part of GM $(4.25 \pm 0.35$ vs. $4.18 \pm$ $0.65 \mathrm{~cm}$, respectively, $p=0.780$ ). Pennation angle and muscle thickness were also similar in the two groups at the medial ( $p=0.519$ and $p=0.216$, respectively) and the distal part of the gastrocnemius ( $p=0.998$ and $p=0.433$, respectively). Ankle angle before stretching was greater in RG compared with VA $\left(120.9 \pm 4.2\right.$ vs. $110.9 \pm 5.8^{\circ}$, respectively, $\left.p=0.001\right)$. During the $1 \mathrm{~min}$ of static stretching, RG displayed greater fascicle elongation compared to the VA at the medial ( $5.86 \pm 0.29$ vs $5.52 \pm 0.53$ $\mathrm{cm}, p=0.048)$ and the distal part ( $6.09 \pm 0.49$ vs $5.15 \pm 0.65 \mathrm{~cm}, p=0.013)$, as well as greater maximal ankle dorsiflexion $(p<0.001)$ and muscle tendon junction displacement $(p<0.001)$. No differences were found between groups in pennation angle $(p>0.458)$ and muscle thickness $(p>0.237)$. CONCLUSIONS: Muscle architectural properties are similar at rest in child athletes with different flexibility backgrounds. However, muscle fascicle elongation is greater in rhythmic gymnasts compared to volleyball athletes and this may contribute to the greater ankle ROM observed in rhythmic gymnasts.
\end{abstract}

Keywords: range of motion; fascicle length; stretching; skeletal muscle ultrasonoraphy; gymnastics; volleyball

(C) 2019 by the authors. Licensee MDPI, Basel, Switzerland. This article is an open access article distributed under the terms and conditions of the Creative Commons Attribution (CC BY) license (http://creativecommons.org/licenses/by/4.0/). 